\title{
Satisfação laboral e a repercussão na qualidade de vida do profissional de enfermagem
}

\section{Job satisfaction and the repercussion in the quality of life of the nursing professional}

Giovana Cópio Vieira ${ }^{1}$, Karina Viana Ribeiro, Aline Ramos Velasco ${ }^{2}$, Érika Almeida Alves Pereira², Elaine Antunes Cortez ${ }^{3,4}$, Joanir Pereira Passos $^{5}$

\section{RESUMO}

Diante da importância do trabalho na vida dos indivíduos, a relação que se estabelece entre o profissional e o ambiente laboral podem implicar repercussões em sua maneira de viver e ser saudável. Não obstante, a qualidade de vida do trabalhador pode estar associada à satisfação profissional, e dentro do contexto da enfermagem, favorecer a uma assistência de qualidade. Portanto, o presente ensaio teve por objetivo identificar publicações sobre satisfação no trabalho e qualidade de vida do profissional de enfermagem; verificar evidências de relação direta entre a satisfação profissional e a qualidade de vida. Esta revisão integrativa compilou artigos através da Base de Dados em Enfermagem (BDENF), Public Medline (PubMed) e o Portal de Periódicos da Coordenação de Aperfeiçoamento de Pessoal de Nível Superior (CAPES). A partir do material analisado, emergiram três categorias temáticas: 1) Fatores que influenciam a satisfação profissional; 2) Importância da satisfação no trabalho e qualidade de vida do profissional para a assistência de enfermagem; 3) Relação do trabalho com a qualidade de vida do trabalhador de enfermagem. Após a análise, não se verificou relação direta entre satisfação no trabalho e qualidade de vida, entretanto, evidenciou-se que insatisfações laborais desencadeiam, potencialmente, problemas físicos e psíquicos, com impacto à qualidade de vida e do serviço prestado.

Palavras-chave: equipe de enfermagem; satisfação no emprego; qualidade de vida; saúde do trabalhador.

\begin{abstract}
In view of the importance of work in individuals' lives, the relationship established between the professional and the work environment may imply repercussions on the way they live and be healthy. Nevertheless, the worker's quality of life may be associated with job satisfaction, and within the context of Nursing, favor quality care. Therefore, the present study objective to identify publications about job satisfaction and quality of life of the nursing professional; to verify evidence of a direct relationship between professional satisfaction and quality of life. The integrative review compiled articles through the Nursing Database (BDENF), Public Medline (PubMed) and the Portal of Periodicals of the Coordination of Improvement of Higher Education Personnel (CAPES). From the material analyzed, three thematic categories emerged: 1) Factors that influence professional satisfaction; 2) Importance of work satisfaction and professional quality of life in nursing care; 3) Relationship of work with quality of life of the nursing worker After the analysis, there was no direct relationship between job satisfaction and quality of life of the professionals, however, it was evidenced that job dissatisfactions trigger, potentially, physical and psychic problems, impacting the quality of life and service provided.
\end{abstract}

Keywords: nursing, team; job satisfaction; quality of life occupational health.

Recebido em: 23/02/2018

Revisado em: 31/05/2018

Aprovado em: 08/06/2018

Autor para correspondência: Giovana Cópio Vieira - Universidade Federal do Estado do Rio de Janeiro - Rua Doutor Xavier Sigaud, 290 - Urca CEP: 22290-180 - Rio de Janeiro (RJ), Brasil - E-mail: giovanavieira@hotmail.com.

Conflito de interesses: nada a declarar. 


\section{INTRODUÇÃO}

Fatores inerentes ao cotidiano profissional da enfermagem, denominados cargas de trabalho, quais sejam; biológicas, químicas, mecânicas, físicas, fisiológicas e psíquicas, relacionam-se, potencialmente, ao comprometimento da qualidade de vida. No hospital, o trabalhador está, simultaneamente, exposto a estas cargas, considerando esse processo como progressivo e cumulativo ${ }^{1}$.

As reflexões acerca do termo qualidade de vida denotam uma noção eminentemente humana, de caráter multidimensional, intimamente associado à saúde, às relações socioambientais, culturais e pessoais ${ }^{2}$. Embora seja de avaliação subjetiva, dependente da carga de conhecimento do sujeito, advinda de suas experiências, valores e da sua história, fala-se em um patamar material mínimo e universal da qualidade de vida. Trata-se, portanto, da satisfação das necessidades mais elementares da vida humana, incluindo valores materiais, dentre eles, o trabalho, e os valores não materiais, como, por exemplo, a realização pessoal ${ }^{3}$.

Ao considerar os elementos que se relacionam com a qualidade de vida, acredita-se que a ocupação profissional, por despender grande parcela de tempo na vida do indivíduo, deva ser considerada uma determinante e a satisfação no desempenho do trabalho está associada a um maior bem estar, realização pessoal e valorização profissional ${ }^{4}$.

Assim como a qualidade de vida, a satisfação profissional apresenta caráter subjetivo, decorrente da percepção do sujeito sobre a sua condição de trabalho. Outrossim, não se trata de uma avaliação estável ou permanente, mas sujeita à análise individual das características do trabalho, aos valores pessoais e da sociedade ${ }^{5}$.

O significado do trabalho para o homem, assim como o tipo de relação que se estabelece entre eles, pode ter implicações na maneira de viver e ser saudável do indivíduo, ou seja, o modo como o trabalhador encontra-se inserido no processo laboral pode refletir em melhor ou pior qualidade de vida ${ }^{2}$.

No processo de trabalho dos profissionais de enfermagem, fatores como as cargas laborais, as condições do trabalho e os riscos ocupacionais, somam-se a oportunidades insuficientes de lazer, reduzido tempo dedicado ao autocuidado e às relações sociais. Este cenário é associado às características ocupacionais, como o regime de plantões, aos múltiplos vínculos, ao intenso ritmo laboral, acompanhado por cargas horárias extensas. Dessa forma, instala-se um contexto favorável ao desgaste, que pode comprometer a qualidade de vida destes trabalhadores ${ }^{5}$.

Observa-se que a enfermagem ainda possui a concepção de sacrifício e autoabandono, desconsiderando as próprias necessidades básicas, pondo os próprios limites físicos e emocionais, em detrimento da sua atividade e função ${ }^{6}$. Não obstante, considera-se improvável proporcionar satisfação aos clientes atendidos, quando os membros da equipe encontram-se insatisfeitos com suas condições, local e a dinâmica de trabalho ${ }^{7}$.

Diante do exposto, este artigo discute e analisa questões envolvidas na qualidade de vida do trabalhador, tendo a satisfação profissional como fator contributivo para ser saudável em seu ofício.
O objetivo foi identificar publicações sobre satisfação no trabalho e a qualidade de vida da equipe de enfermagem e verificar se há evidências de relação direta entre a satisfação no trabalho e a qualidade de vida destes profissionais.

\section{MÉTODOS}

Revisão integrativa de literatura, cuja finalidade é auxiliar nas discussões relacionadas aos métodos e resultados de pesquisas, permitindo reflexões que subsidiem estudos futuros, a partir de padrões metodológicos rigorosos ${ }^{8}$.

Utilizou-se o banco de dados da Biblioteca Virtual em Saúde (BVS), selecionando a Base de Dados em Enfermagem (BDENF), Public Medline (PubMed) e o Portal de Periódicos da Coordenação de Aperfeiçoamento de Pessoal de Nível Superior (CAPES).

A revisão integrativa é realizada em seis etapas: seleção de hipóteses ou questão de pesquisa; seleção dos estudos que comporão a amostra; categorização dos estudos; análise dos achados da revisão; interpretação dos resultados; e síntese com as principais evidências, proporcionando exame crítico dos achados ${ }^{8}$.

Na primeira etapa elaborou-se a questão norteadora: “Quais são as evidências científicas sobre a satisfação no trabalho e sua relação com a qualidade de vida da equipe de enfermagem"?

Na segunda etapa, se estabeleceu critérios de inclusão: artigos no idioma português, inglês ou espanhol, publicados entre 2010 a 2015, que abordassem a satisfação profissional e a qualidade de vida na equipe de enfermagem. Como critérios de exclusão: trabalhos científicos não oriundos de pesquisa original, realizados fora do ambiente hospitalar e que envolvessem outros profissionais.

A pesquisa utilizou a estratégia PICO, qual representa um acrônimo para Paciente, Intervenção, Comparação e Outcomes, palavra que se refere ao desfecho encontrado. Tais componentes são estruturais para a construção da questão de estudo, visando obter resultados que sirvam de esteio à Prática Baseada em Evidências $(\mathrm{PBE})^{9}$. Porém, neste estudo não houve comparação, sendo utilizado, desta forma, o PIO, onde o P - (paciente/problema), I - (intervenção), $\mathrm{O}$ - (resultado/desfecho).

Foram escolhidos os seguintes Medical Subject Headings $(\mathrm{MeSH})$ e seus respectivos Descritores em Ciências da Saúde (DeCS): para P- "Nursing, Team" ("Equipe de Enfermagem") "or" "Nursing” ("Enfermagem”); para I- "Job Satisfaction” ("Satisfação no Emprego"), e para O- "Quality of Life” (“Qualidade de vida”) "or" "Occupational Health" (“Saúde do trabalhador”). Os descritores componentes de cada item da PIO foram combinados através do operador booleano "and". A coleta de dados ocorreu em setembro de 2016.

Na terceira etapa 147 artigos foram coligidos e filtrados no recorte temporal, resultando em 81 artigos que passaram por refino de critérios, até se obter uma amostra final constituída por sete artigos, sendo três obtidos no PubMed, dois na BDENF e dois no Portal CAPES. O curso da busca e seleção da amostra está representado na Figura 1. 
$\mathrm{Na}$ quarta etapa, os textos compilados foram analisados a partir da qualidade metodológica, do seu nível de evidência, e da relevância dos resultados.

Destaca-se que a Prática Baseada em Evidências (PBE) possibilita a tomada de decisão sobre questões relacionadas à assistência à saúde, a partir do consenso de evidências ${ }^{10}$. Nesta pesquisa será utilizado o nível de evidência de acordo com o Joanna Briggs Institute (JBI), agência reconhecida por fomentar pesquisas sobre cuidados de saúde baseados na utilização de evidências, classificadas por meio dos critérios de viabilidade, significação, adequação e eficácia ${ }^{11}$.

$\mathrm{Na}$ quinta etapa, os resultados foram interpretados e discutidos, comparando os dados evidenciados com o conhecimento teórico. Destacaram-se os trabalhos que abordavam objetivamente a satisfação no trabalho e sua repercussão na qualidade de vida.
Como última etapa, o material obtido foi explorado através de leitura intensiva, buscando-se encontrar ideias significativas, que se repetiam ou que se mostrassem divergentes no que concerne à satisfação profissional e qualidade de vida do trabalhador de enfermagem. Assim, procedeu-se a interpretação e síntese do conteúdo dos artigos pesquisados, emergindo características consideradas elementos-chave, agrupadas em três categorias temáticas, de modo que fossem comparáveis dentro do contexto da satisfação laboral, da qualidade de vida e do trabalho de enfermagem.

\section{RESULTADOS}

A seguir, o Quadro 1 contém as publicações selecionadas com suas respectivas referências relacionadas ao título, autor, ano

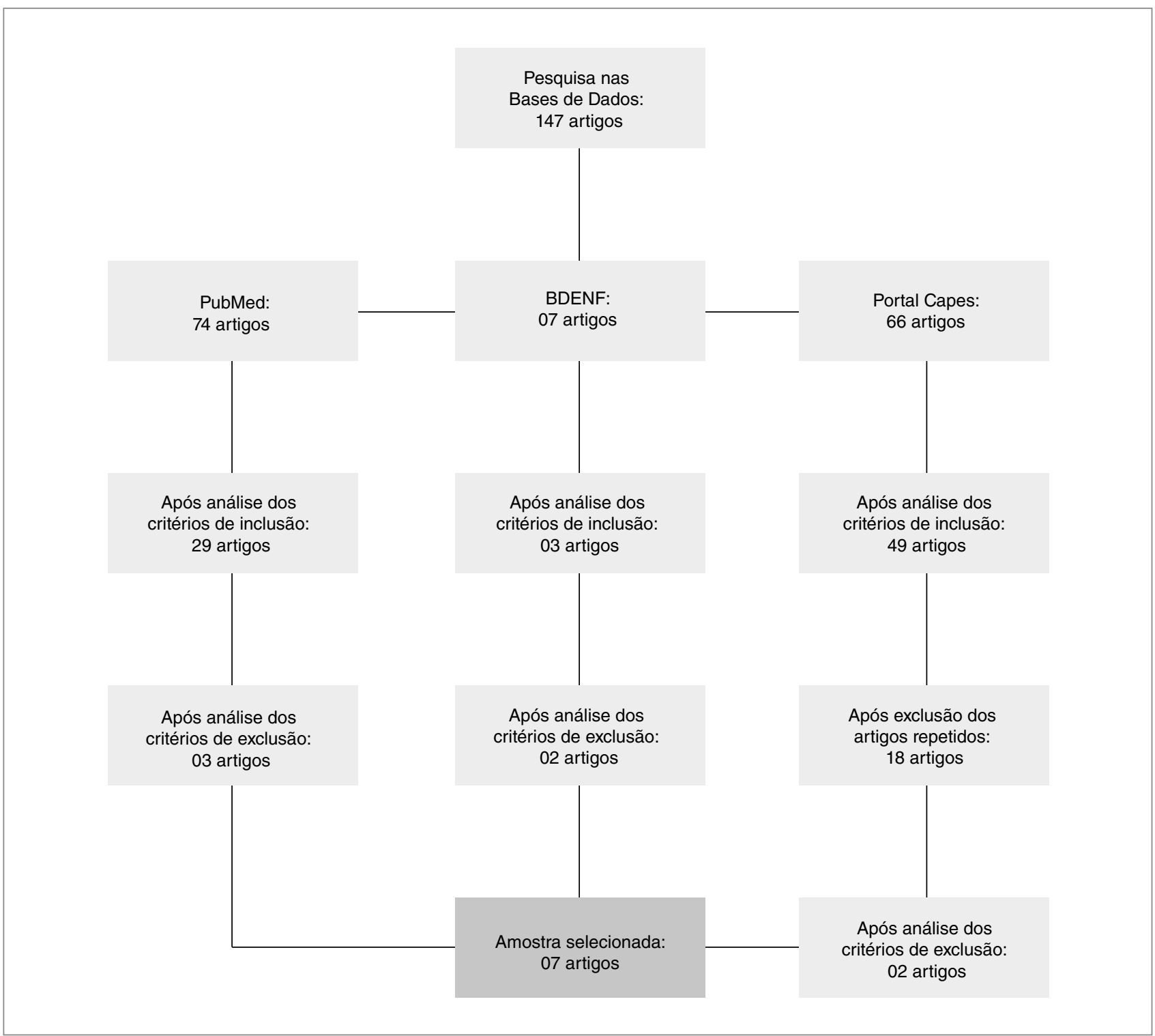

Figura 1: Fluxograma do processo de seleção dos artigos, segundo as bases de dados. 
assim como ao país de publicação, metodologia, nível de evidência (NE) e objetivos.

Os resultados organizam-se em categorias temáticas (Quadro 2), quais sejam: 1) fatores que influenciam a satisfação profissional; 2) importância da satisfação no trabalho e qualidade de vida do profissional para a assistência de enfermagem 3) relação do trabalho com a qualidade de vida do trabalhador de enfermagem.

Os estudos de Kudo et al. ${ }^{12}$, Lemos et al. ${ }^{13}$, Renner et al. ${ }^{14}$, Atefi et al. ${ }^{15}$ discorrem na primeira categoria temática; os estudos de Arakawa et al. ${ }^{16}$, Lemos et al. ${ }^{13}$, Van Bogaert et al. ${ }^{17}$, na segunda. Já Souza et al. ${ }^{18}$ versam sobre a influência do trabalho na qualidade de vida e Renner et al. ${ }^{14}$ sobre a satisfação profissional na qualid de de vida, portanto, foram agrupados na terceira categoria (Quadro 2).

\section{DISCUSSÃo}

\section{Fatores que influenciam a satisfação profissional}

Os fatores mais frequentemente relacionados à satisfação profissional foram: satisfação com salário, tempo livre para fazer suas próprias atividades, o envolvimento com o cuidado do paciente que gera um sentimento espiritual de satisfação, melhoria de habilidades e do desempenho profissional, o trabalho em equipe e a participação na tomada de decisões ${ }^{12-15}$.

Já as causas de insatisfação foram associadas ao ambiente físico (falta de condições adequadas de trabalho, de recursos, escassez de material e de profissionais); ao ambiente psicossocial (conflitos com a chefia, ausência de clareza na distribuição de tarefas e responsabilidades, desunião da equipe, jornada extensa, rigidez organizacional, sem benefícios/recompensas, discriminação pelo papel desempenhado, habilidade de liderança deficiente e remuneração insatisfatória). Destacam-se as queixas de baixa remuneração, qual exigiria mais horas de trabalho, à custa da restrição no tempo destinado ao lazer, repouso e contato com a família o que pode ocasionar problemas de saúde tanto no âmbito físico quanto psicossocial ${ }^{12-15}$.

A Organização Mundial de Saúde (OMS) levanta exemplos de perigos psicossociais, dentre eles inclui uma organização do trabalho deficiente, a falta de apoio para um equilíbrio entre a vida profissional e familiar, além do estilo de administração adotado, em que uma gerência sem negociações, a ausência de feedbacks construtivos e a falta de gestão do desempenho de forma respeitosa tornam-se riscos para as relações psicossociológicas ${ }^{19}$.

Quadro 1: Dados das publicações selecionadas no período de 2010 a 2015.

\begin{tabular}{|c|c|c|c|c|}
\hline Referência & País & Metodologia & $\begin{array}{l}\text { Nível de } \\
\text { evidência }\end{array}$ & Objetivos \\
\hline $\begin{array}{l}\text { Work motivation for Japanese nursing } \\
\text { assistants in small- to medium-sized } \\
\text { hospitals. Kudo et al. } .^{12}\end{array}$ & Japão & $\begin{array}{l}\text { Quantiqualitativo. Amostra: } \\
516 \text { auxiliares de enfermagem. } \\
\text { Instrumento: Questionário. }\end{array}$ & IV & $\begin{array}{l}\text { Examinar preditores significativos } \\
\text { associados ao trabalho de auxiliares de } \\
\text { enfermagem e a satisfação relacionada. }\end{array}$ \\
\hline $\begin{array}{l}\text { Satisfação no trabalho da enfermagem } \\
\text { em UTI. Lemos et al. } .^{13}\end{array}$ & Brasil & $\begin{array}{l}\text { Qualitativa. Amostra: } 30 \\
\text { profissionais da equipe de } \\
\text { enfermagem. Instrumento: } \\
\text { Entrevista Semi-estruturada. }\end{array}$ & IV & $\begin{array}{c}\text { Identificar e classificar as } \\
\text { expressões de satisfação no trabalho } \\
\text { da equipe de enfermagem. }\end{array}$ \\
\hline $\begin{array}{l}\text { Qualidade de vida e satisfação no } \\
\text { trabalho: a percepção dos técnicos de } \\
\text { enfermagem que atuam em ambiente } \\
\text { hospitalar. Renner et al. }{ }^{14}\end{array}$ & Brasil & $\begin{array}{c}\text { Quantiqualitativa } \\
\text { Amostra: } 49 \text { técnicos } \\
\text { de enfermagem. } \\
\text { Instrumento: Questionário de } \\
\text { Design Macroergonômica. }\end{array}$ & IV & $\begin{array}{l}\text { Verificar a percepção dos técnicos de } \\
\text { enfermagem sobre sua qualidade de vida } \\
\text { sob o aspecto da satisfação com o trabalho; } \\
\text { Investigar as características ergonômicas } \\
\text { do trabalho sob a ótica da macroergonomia } \\
\text { e as condições de trabalho. }\end{array}$ \\
\hline $\begin{array}{l}\text { Factors influencing registered nurses } \\
\text { perception of their overall job satisfaction: } \\
\text { a qualitative study. Atefi et al. }{ }^{15}\end{array}$ & Malásia & $\begin{array}{l}\text { Qualitativo. Amostra: } 85 \\
\text { enfermeiros em um hospital do Irã. } \\
\text { Instrumento: Entrevistas } \\
\text { semi-estruturada. }\end{array}$ & IV & $\begin{array}{l}\text { Explorar fatores relacionados à } \\
\text { assistência de enfermagem à pacientes } \\
\text { críticos com a satisfação ou insatisfação } \\
\text { no trabalho dos enfermeiros no Irã. }\end{array}$ \\
\hline $\begin{array}{l}\text { Factors contributing to medical errors } \\
\text { and incidents among hospital nurses } \\
\text {--nurses' health, quality of life, and } \\
\text { workplace predict medical errors and } \\
\text { incidents. Arakawa et al. }{ }^{16}\end{array}$ & Japão & $\begin{array}{l}\text { Quantiqualitativo. Amostra: } \\
6.445 \text { enfermeiras. } \\
\text { Instrumento: Questionário. }\end{array}$ & IV & $\begin{array}{l}\text { Examinar a correlação de incidentes } \\
\text { médicos e erros entre enfermeiros } \\
\text { com fatores de estilo de vida, saúde e } \\
\text { ambiente de trabalho. }\end{array}$ \\
\hline $\begin{array}{l}\text { Nursing unit teams matter: Impact of } \\
\text { unit-level nurse practice environment, } \\
\text { nurse work characteristics, and burnout } \\
\text { on nurse reported job outcomes, and } \\
\text { quality of care, and patient adverse } \\
\text { events - A cross-sectional survey. Van } \\
\text { Bogaert et al. }{ }^{17}\end{array}$ & Bélgica & $\begin{array}{l}\text { Quantitativo. Amostra: } \\
1108 \text { enfermeiros. } \\
\text { Instrumento: Questionário. }\end{array}$ & IV & $\begin{array}{l}\text { Investigar o impacto dos fatores } \\
\text { ambientais, das características do } \\
\text { trabalho e burnout como resultados } \\
\text { da prática de trabalho de enfermeiras, } \\
\text { qualidade do cuidado e eventos } \\
\text { adversos em pacientes como variáveis } \\
\text { ao nível da unidade de enfermagem. }\end{array}$ \\
\hline $\begin{array}{l}\text { Influência do turno de trabalho e cronotipo } \\
\text { na qualidade de vida dos trabalhadores de } \\
\text { enfermagem. Souza et al. }{ }^{18}\end{array}$ & Brasil & $\begin{array}{l}\text { Quantitativo. Amostra: } 101 \\
\text { sujeitos - equipe de enfermagem. } \\
\text { Instrumento: Questionário } \\
\text { Matutinidade-Vespertinidade } \\
\text { e WHOWOLbreve. }\end{array}$ & IV & $\begin{array}{l}\text { Verificar a influência do turno de trabalho } \\
\text { e cronotipo na qualidade de vida dos } \\
\text { trabalhadores de um hospital universitário. }\end{array}$ \\
\hline
\end{tabular}


De acordo com a "Teoria dos Dois Fatores" de Frederick Herzberg, a insatisfação é determinada pela deficiência em fatores extrínsecos ou higiênicos. Por outro lado, os aspectos agradáveis e satisfatórios dizem respeito aos fatores intrínsecos ou motivadores ${ }^{20}$.

Dessa forma, a satisfação no trabalho é associada a elementos que promovem o desenvolvimento pessoal, reconhecimento e autorrealização, relacionados ao desempenho de experiências desafiadoras, da responsabilidade exigida e ao conteúdo do cargo. Já os elementos extrínsecos, são assim denominados por serem externos ao indivíduo, estando relacionados aos aspectos do trabalho, como a supervisão, o salário, as condições e o ambiente de trabalho, assim como a segurança no emprego ${ }^{12}$.

Sob a perspectiva da relação entre a ocupação laboral e a qualidade de vida, reconhece-se que o trabalho está relacionado ao melhor bem estar, à satisfação pessoal e profissional. Desta maneira, é necessário que o homem se realize naquilo que faz, sinta-se satisfeito com a atividade escolhida, de modo que a mesma possa atender às expectativas de cada um.

Para melhorar a satisfação profissional deveria se oferecer oportunidades de desenvolvimento profissional, além de salários baseados em desempenho, especialidade, política de incentivos e outros benefícios ${ }^{10}$. Além disso, gestores e administradores das instituições devem monitorar as condições, assim como a carga e o ambiente de trabalho de seus funcionários, considerando sua qualidade de vida ${ }^{12}$

\section{Importância da satisfação no trabalho e qualidade de vida do profissional para a assistência de enfermagem}

Nesta categoria, discute-se o impacto da satisfação no trabalho e da qualidade de vida na prática do cuidado, ressaltando que os problemas emocionais e físicos como dor corporal, a quantidade de afastamentos para tratamento de saúde dentro de uma equipe, ambiente de trabalho e plantões noturnos podem favorecer a ocorrência de incidentes/erros na assistência ${ }^{16}$. Ademais, o convívio conflituoso dentro do trabalho, devido à falta de apoio e de relações respeitosas é importante causas de esgotamento, gerando níveis elevados de Burnout e resultados indesejados na qualidade do atendimento ${ }^{17}$, visto que os membros da equipe podem reproduzir suas insatisfações na relação de cuidado com o paciente, podendo prejudicá-lo7.

As insatisfações no trabalho, níveis elevados de Burnout, o acometimento por doença durante o turno de trabalho, carga horária excessiva, turno noturno sem pausas, problemas administrativos, assim como a sobrecarga de tarefas é variável associada a maior frequência de reclamações e conflitos entre profissionais, pacientes e familiares, maior ocorrência de erros ou incidentes, resultados indesejados nos cuidados prestados, baixa qualidade da assistência e insatisfação do paciente ${ }^{17}$.

Para assegurar uma assistência eficiente é necessário que os integrantes da equipe de enfermagem encontrem-se satisfeitos com o trabalho que realizam, para que seu desempenho ao cuidar dos clientes possa estar integralmente desenvolvido ${ }^{7}$.

Investir na saúde do trabalhador é investir na qualidade da assistência. Portanto, avaliar a exaustão emocional e física deste profissional e sua qualidade de vida, pode ser uma alternativa para identificar causas de queixas de pacientes e de queda na produtividade e qualidade da assistência. Estudos dentro da unidade hospitalar, centrados na saúde física e mental do profissional podem melhorar o atendimento.

\section{Relação do trabalho e da satisfação profissional com a qualidade de vida do trabalhador de enfermagem}

A partir do perfil cronobiológico do trabalhador, questiona-se o impacto do turno de trabalho na qualidade de vida, não havendo

Quadro 2: Principais resultados das publicações de 2010 a 2015, conforme os eixos temáticos.

\begin{tabular}{|c|c|}
\hline $\begin{array}{l}1^{\text {a }} \text { Categoria: Fatores que } \\
\text { influenciam a satisfação profissional }\end{array}$ & $\begin{array}{c}2^{\text {a }} \text { Categoria: Importância da satisfação no } \\
\text { trabalho e qualidade de vida do profissional } \\
\text { para a assistência de enfermagem }\end{array}$ \\
\hline $\begin{array}{l}\text { - Motivação }{ }^{12,15} \\
\text { - Tempo livre para realizar tarefas da vida pessoal }{ }^{12} \\
\text { - Reconhecimento e relacionamentos } \\
\text { interpessoais }^{12,13,14} \\
\text { - Participação na tomada de decisão } \\
\text { - Progressos nas habilidades de trabalho }{ }^{12} \\
\text { - Remuneração não é motivação principal }{ }^{12} \\
\text { - Valorização da natureza intrínseca e } \\
\text { experiência do trabalho }{ }^{12} \\
\text { - Remuneração satisfatória }{ }^{13,14} \\
\text { - Boas condições de trabalho }{ }^{13} \\
\text { - Valorização profissional } \\
\text { - Ambiente social confortável } \\
\text { - Ambiente físico de trabalho adequado } \\
\text { - Sentimento espiritual }{ }^{13}\end{array}$ & $\begin{array}{l}\text { - Incidentes/erros mais frequentes entre } \\
\text { enfermeiras que apresentavam dor corporal, } \\
\text { problemas emocionais durante o trabalho e } \\
\text { nas acometidas por doenças nos últimos } 6 \\
\text { meses anteriores aos erros }^{16} \text {. } \\
\text { - A falta de descanso no turno de trabalho noturno } \\
\text { gera maior frequência de erros na assistência }{ }^{16} \text {. } \\
\text { - A equipe de enfermagem reconhece } \\
\text { que a satisfação no trabalho gera } \\
\text { bom resultado ao cliente }{ }^{13} \text {. } \\
\text { - Gestão de enfermagem, capital social, } \\
\text { exaustão emocional e despersonalização } \\
\text { são preditores que interferem nas seguintes } \\
\text { variáveis: insatisfação no trabalho, intenção } \\
\text { de mudança de emprego e qualidade dos } \\
\text { cuidados prestados }{ }^{17} \text {. } \\
\text { - Fatores ambientais, características do trabalho } \\
\text { e níveis de burnout dos profissionais foram } \\
\text { associados a maior frequência de reclamações } \\
\text { de pacientes e familiares, quedas de pacientes, } \\
\text { infecções hospitalares e erros de medicação }{ }^{17} \text {. }\end{array}$ \\
\hline
\end{tabular}

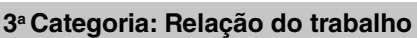 com a qualidade de vida do trabalhador de enfermagem}

- Não houve associação direta entre turno de trabalho e qualidade de vida. Entretanto, houve associação significativa entre concordância do cronotipo com o turno de trabalho e a qualidade de vida no domínio meio ambiente. Ou seja, sujeitos que apresentaram cronotipo (matutino, vespertino ou noturno) concordante com o turno de trabalho, apresentaram escores melhores no domínio meio ambiente da qualidade de vida. Este domínio relacionase aos aspectos estruturais da vida, como oportunidade de recreação/lazer, recursos financeiros, oportunidade de adquirir novas informações e habilidades, ambiente físico, disponibilidade e qualidade dos cuidados de saúde e sociais, segurança física e proteção. Sugerindo que esta concordância seja um fator protetor de qualidade de vida ${ }^{18}$.

- Convívio familiar, lazer e descanso restrito decorrente de longa jornada de trabalho implica em sobrecarga física e emocional, interferindo negativamente na qualidade de vida $^{14}$. 
evidência de associação entre estas duas variáveis ${ }^{18}$. Já o descuido do profissional quanto ao seu bem-estar e qualidade de vida, foi apontado como fator de impacto negativo a sua saúde ${ }^{14}$. Não obstante, mantém-se envolvido no cuidado, proporcionando bem-estar e melhora na qualidade de vida de terceiros.

Sabe-se, todavia, da influência negativa do trabalho noturno à saúde e bem-estar dos trabalhadores em quatro esferas: 1) a biológica, causando distúrbios no ritmo biológico; 2) a social, com as dificuldades para manter um relacionamento com a sociedade e família, com consequências negativas ao matrimônio, cuidado às crianças e contatos sociais; 3 ) a médica, com problemas para sua saúde, que podem se manifestar em distúrbios do sono e de hábitos alimentares; 4) e no trabalho, onde pode levar a erros e acidentes ${ }^{21}$.

A dissociação na relação entre trabalho noturno e a qualidade de vida pode ser atribuída à conveniência (adicional noturno, autonomia, possibilidade de segundo emprego e/ou de continuar estudando) sobreposta aos prejuízos descritos ${ }^{18}$.

Independente do turno, doenças ocupacionais podem surgir ao longo dos anos, com manifestações, tanto de ordem física, que são: enxaquecas, irritação, desgaste físico, dores nas pernas, varizes, problemas de coluna, hipertensão arterial, etc.; quanto psíquica, que são: depressão, estresse, desarranjos no seio familiar, transtornos mentais comuns, distúrbios alimentares e do sono, alterações no estado de atenção e alerta, assim como neuroses que, muitas vezes, levam a acidentes de trabalho e a licenças ${ }^{22}$.

Assim, a OMS contribui para a compreensão da saúde ocupacional, centrada no ambiente físico de trabalho, não deixando de incluir os fatores psicossociais, assim como as necessidades de práticas de saúde individual. $\mathrm{O}$ ambiente de trabalho surge como um espaço para o incentivo das atividades preventivas e do cuidado com a saúde considerando o fato não só para evitar doenças e acidentes de trabalho, mas com o propósito de diagnosticar e melhorar a saúde em geral ${ }^{19}$.

\section{CONCLUSÃO}

As condições do ambiente físico e psicossocial do trabalho, a carga horária, a remuneração, o desenvolvimento de habilidades, o desempenho e o reconhecimento profissional, assim como o tempo dedicado ao lazer, família e tarefas fora do trabalho são os aspectos que mais influenciam a satisfação dos profissionais de enfermagem.

Não foi possível verificar uma relação direta entre a satisfação no trabalho e a qualidade de vida do profissional a partir das publicações, pois há parcos estudos que analisam, concomitantemente, as duas variáveis. Entretanto, há evidências de que as insatisfações no trabalho podem desencadear manifestações físicas e psíquicas, propiciando o surgimento de doenças ocupacionais, acidentes de trabalho e licenças para tratamento de saúde, impactando diretamente na qualidade de vida do profissional, outrossim, na qualidade do serviço prestado.

Mais pesquisas contribuirão para o desenvolvimento de políticas voltadas à criação de ambientes e condições de trabalho saudáveis, trazendo à luz os conceitos de qualidade de vida no trabalho e qualidade de vida dos profissionais da saúde em geral.

\section{REFERÊNCIAS}

1. Mininel VA, Baptista PCP, Felli VEA. Psychic workloads and strain processes in nursing workers of brazilian university hospitals. Rev Latino-Am. Enfermagem. 2011;19(2):340-7 http://dx.doi.org/10.1590/S0104-11692011000200016

2. Cecagno D, Gallo CMC, Cecagno S, Siqueira HCH. Qualidade de vida e o trabalho sob a ótica do enfermeiro. Cogitare Enferm. 2002;7(2):54-9. http://dx.doi.org/10.5380/ce.v7i2.1669

3. Minayo MCS, Hartz ZMA, Buss PM. Qualidade de vida e saúde: um debate necessário. Ciênc Saúde Coletiva. 2000;5(1):7-18.

4. Haddad MCL. Qualidade de vida dos profissionais de enfermagem. Rev Espaç Saúde. 2000;1(2):75-88.

5. Vieira GC. O papel da satisfação profissional na qualidade de vida do enfermeiro. Dissertação (Mestrado). Universidade Federal do Estado do Rio de Janeiro. Rio de Janeiro:2017.

6. Pizzoli LML. Qualidade de vida no trabalho: um estudo de caso das enfermeiras do Hospital Heliópolis. Ciênc Saúde Coletiva. 2005;10(4):1055-62.

http://dx.doi.org/10.1590/S1413-81232005000400028

7. Regis LFLV, Porto IS. Necessidades humanas básicas dos profissionais de enfermagem: situações de (in)satisfação no trabalho. Rev Esc Enferm USP. 2011;45(2):334-41. http://dx.doi.org/10.1590/S0080-62342011000200005
8. Mendes KDS, Silveira RCCP, Galvão CM. Revisão Integrativa: método de pesquisa para a incorporação de evidências na saúde e na enfermagem. Texto Contexto Enferm. 2008;17(4):758-64.

http://dx.doi.org/10.1590/S0104-07072008000400018

9. Santos CMC, Pimenta CAM, Nobre MRC. The PICO strategy for the research question construction and evidence search. Rev Latino-Am Enfermagem. 2007;15(3):508-11.

http://dx.doi.org/10.1590/S0104-11692007000300023

10. Driever MJ. Are evidence-based practice and best practice the same? West J Nurs Res. 2002;24(5):591-7. http://dx.doi.org/10.1177/019394502400446342

11. Karino ME, Felli VEA. Enfermagem baseada em evidências: avanços e inovações em revisões sistemáticas. Cienc Cuid Saude. 2012:11(Supl.):11-15.

http://dx.doi.org/10.4025/cienccuidsaude.v11i5.17048

12. Kudo $Y$, Kido S, Shahzad T, Yoshimura E, Shibuya A, Aizawa Y. Work motivation for Japanese nursing assistants in small- to medium-sized hospitals. Tohoku $\mathrm{J}$ Exp Med. 2011;225(4):293-300

13. Lemos MC, Rennó CO, Passos JP. Satisfação no trabalho da enfermagem em UTI. Rev Pesq Cuid Fundam. 2012;4(4):2890-2900. 
14. Renner JS, Taschetto DVR, Baptista GL, Basso CR. Qualidade de vida e satisfação no trabalho: a percepção dos técnicos de enfermagem que atuam em ambiente hospitalar. Rev Min Enferm. 2014;18(2):440-6.

http://www.dx.doi.org/10.5935/1415-2762.20140033

15. Atefi N, Abdullah K.L, Wong LP, Mazlom R. Factors influencing registered nurses perception of their overall job satisfaction: a qualitative study. Intern Nurs Rev. 2014;61(3):352-60. http://www.dx.doi.org/10.1111/inr.12112

16. Arakawa $C$, Kanoya $Y$, Sato $C$. Factors contributing to medical errors and incidents among hospital nurses - nurses' health, quality of life, and workplace predict medical errors and incidents. Ind Health. 2011;49(3):381-8. http://dx.doi.org/10.2486/indhealth.MS968

17. Van Bogaert $P$, Timmermans $O$, Weeks SM, van Heusden $D$, Wouters K. Franck E. Nursing unit teams matter: Impact of unitlevel nurse practice environment, nurse work characteristics, and burnout on nurse reported job outcomes, and quality of care, and patient adverse events-A cross-sectional survey. Int J Nurs Stud. 2014;51(8):1123-34

http://dx.doi.org/10.1016/j.ijnurstu.2013.12.009
18. Souza SBC, Tavares JP, Macedo ABT, Moreira PW, Lautert L. Influência do turno de trabalho e cronotipo na qualidade de vida dos trabalhadores de enfermagem. Rev Gaúcha Enferm. 2012;33(4):79-85

http://dx.doi.org/10.1590/S1983-14472012000400010

19. Organização Mundial da Saúde (OMS). Ambientes de trabalho saudáveis: um modelo para ação: para empregadores, trabalhadores, formuladores de política e profissionais. Brasília: SESI; 2010

20. Dias MFFG, Proença MTVC. A motivação dos enfermeiros em tempo de crise. Dissertação (Mestrado) - Faculdade de Economia Universidade do Porto. Porto: 2012

21. Silva RM, Beck CLC. Satisfação profissional dos enfermeiros de um hospital universitário no trabalho noturno. Dissertação (Mestrado) - Universidade Federal de Santa Maria. Santa Maria: 2008.

22. Reis LC, Belmont LG, Taveira TMC, Cortez EA, Avanci BS Os fatores do processo de trabalho da enfermeira que interferem na sua qualidade de vida. Rev Pesq Cuid Fund. 2010;2(1):489-502. 